\title{
The elastic local buckling behaviour and strength of the simply supported I-beam utilising the energy equilibrium method
}

\author{
S.M. Fujak, Y. Kimura \& A. Suzuki \\ Department of Architecture and Building Science, Tohoku University, Sendai, Miyagi, Japan
}

\begin{abstract}
One of the phenomena influencing the capacity of the structure, or the ultimate state, is local buckling of the frame's members. This article presents the analyses of the most common setup, the simply supported beam with an I-section, and proposes the equations to estimate the local buckling strength of such beam. The derivation of the solution is based upon the principles of the theory of elastic stability, the shell stability, and the equilibrium of the work done by external forces and the plate's strain energy. Solutions are proposed for the segments of the beam, depending on the geometrical properties of those, and are later connected into equations considering the coupled interactions between the web and flanges. Finally, the equation allowing for the precise estimation of the strength of the whole beam is proposed.
\end{abstract}

\section{INTRODUCTION}

\subsection{Previous research}

Local buckling is one of the common forms of the stability loss occurring within the section, without the lateral displacement or twist of the whole beam. Local buckling has a significant influence on the behaviour of the whole structure, drastically reducing the frame's capacity or leading to global loss of stability i.e. the collapse of the structure (Fujak \& Kimura 2019).

Full understanding of the local buckling phenomenon allows the engineer to correctly approximate the ultimate state of the structure, securing the linear behaviour in the elastic stage and nonlinear behaviour influenced only by the material properties in the plastic stage. Moreover, it allows for the correct classification of the section and determination if the members can stand the yielding load (sections class 1-3, see Eurocode 1993-1-1 (2005)), or are endangered by the local stability loss in the elastic work range (class 4). Additionally, the correct method of the analyses may be determined by the members elastic buckling strength. $\alpha_{c r}$ is the value of the elastic critical buckling load for the global instability mode based on initial elastic stiffness, $F_{c r}$, divided by the design loading on the structure, $F_{E d}$. Eurocode 1993-1-1 (2005) allows for the use of the first-order analysis only when $\alpha_{c r}$ is higher than 10 for the elastic analyses and 15 for plastic analyses. Therefore, understanding of the elastic buckling strength of the members is crucial to correctly calculate the capacities of the structure.

Previous research on local bucking capacity is based upon the Euler-Bernoulli beam theory and Kirchhoff-Love plate theory. Timoshenko \& Gere (1961) proposed the solutions for plates with simple boundary conditions and certain aspect ratios. With limited access to solving tools however, not all the solutions were given, and the effects of the flange-web interactions were not analysed. Roberts \& Jhita (1983) analysed the problem of the elastic buckling of the I-beam focusing mostly on the strength of the flange. Bradford \& Hancock (1984) described the method to consider the uneven stress distribution in wide-flange sections in the post-buckling stage.

Most of the recent research work is related to the problem of elastoplastic bucking. Ikarashi (2003) proposed the method of estimating the elastic buckling capacity of the whole beam

DOI: $10.1201 / 9781003132134-53$ 
based on the buckling capacity of the web. However, this research was conducted to explain the plastic behaviour of the beam, so the precision of results in the elastic stage is moderate. Later works (Ikarashi et al. 2011, Kimura et al. 2019) proposed the indexes for the beam classification with respect to both length of the beam and loading applied to the element. These researches, however, focus on the capacity of the elements in the plastic stage.

To overcome the previously mentioned limitations research on the elastic buckling capacity of the simply supported beams is necessary. The aim of this study is:

- To investigate the strength of the plates (web, flanges) - segments building up the simply supported beam depending on their aspect ratios, with the effects of interaction between the segments considered.

- To propose simplified equations allowing for the estimation of local buckling capacity of the simply-supported beam.

\subsection{Research proposal}

The research presented analyses the elastic buckling strength of simply supported I-beams. Two types of commonly used loading are used - point load (hereinafter PL) and uniformly distributed load (UDL). The beam is as presented in Figure 1. Figure 1a presents the beam loaded with PL and the resultant stresses in the segments of the beam - the web and the flange. In the same manner, Figure $1 \mathrm{~b}$ presents the loading and stress distribution in the beam loaded with UDL. Segments presented have simplified boundary conditions (BC) - either pinned or fixed. In reality, $\mathrm{BC}$ of the separate segments are semi-stiff, as the stiffness of the web influences the rotation of the flange and vice-versa. Note that the beams are ended with stiff diaphragm, which control node has certain degrees of freedom blocked $\left(\delta_{x}, \delta_{y}, \delta_{z}, \varphi_{x}, \varphi_{z}\right.$ in case of the left-hand side end and $\delta_{y}, \delta_{z}, \varphi_{x}, \varphi_{z}$ in case of the right-hand side end). The stresses and deformations of the beam/plates are symmetric about the vertical axis of the beam. $x, y, z$ are axes, $L$ stands for the half of the length of the beam/plate, $P_{i}$ for the PL in the $i$ direction, $q_{i}$ for the UDL in the $i$ direction, $M_{i}$ for the bending moment around the $i$ axis $\delta_{i}$ for the deflection in the $i$ direction, $\varphi_{i}$ for the rotation around the $i$ axis, $\sigma_{i}(j, k)$ for the axial

a)

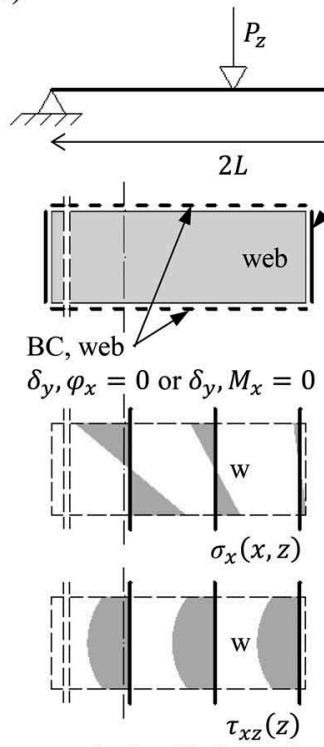

(web with flanges)
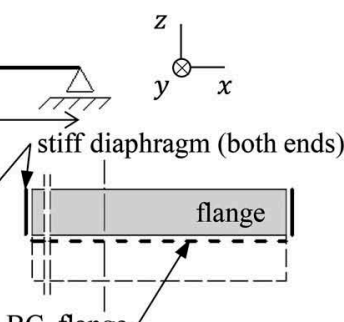

$\mathrm{BC}$, flange
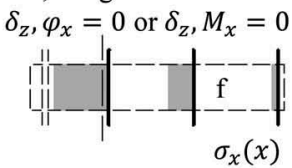

$\left(\tau_{x z} \cong 0\right)$

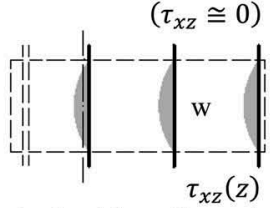

(web without flanges) b)

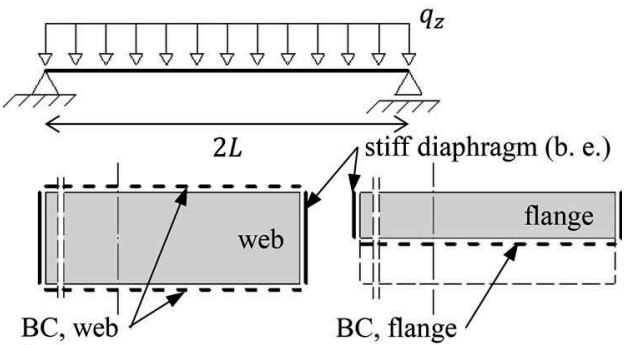

$\delta_{y}, \varphi_{x}=0$ or $\delta_{y}, M_{x}=0$

$\delta_{z}, \varphi_{x}=0$ or $\delta_{z}, M_{x}=0$
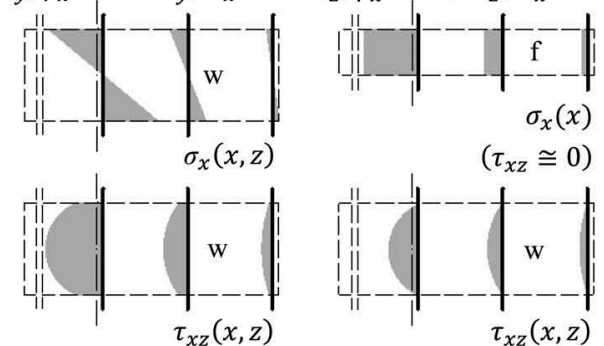

(web with flanges) $\left(\tau_{x z} \cong 0\right)$

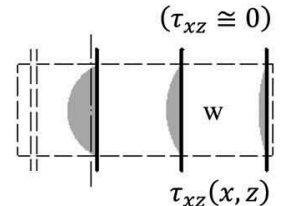

(web without flanges)

Figure 1. Simply supported beam - analysed BCs, loads and stresses. a) Simply supported beam loaded with PL, b) Simply supported beam loaded with UDL. 


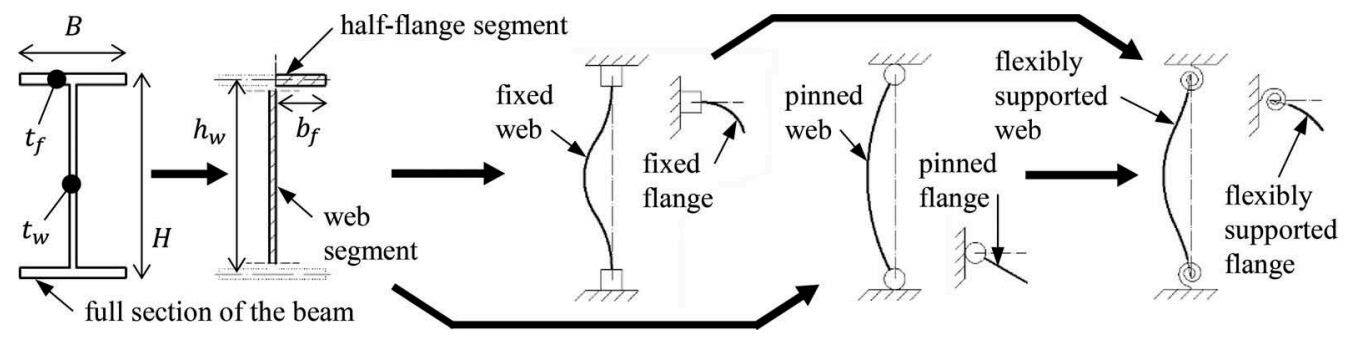

Figure 2. Beam's section and segments' BC.

stress in the $i$ direction as a function of $j$ and $k$ coordinates and $\tau_{i, j}(k, l)$ for the shear stress in the $i, j$ plane).

In order to analyse the section, it is crucial to understand the local behaviour of its segments. Therefore, the beam is first separated into segments, web and flanges, with simplified BC (Figure 2). The analyses of the detailed BC of web/flange influence is addressed in section 4.

As the flange is symmetrical about the vertical axis, it can be assumed to be built of two identical half-flanges. Note that $B$ stands for the beam width, $H$ for the height, $b_{f}$ for the halfflange width, $h_{w}$ for the web height, $t_{f}$ for the flange thickness and $t_{w}$ for the web thickness.

The proposed research is based on the energy equilibrium between the work done by external forces and the strain energy of the plate, see (1-4) (Timoshenko \& Gere 1961).

$$
\begin{gathered}
\Delta T=\Delta U \\
\Delta T=-\frac{1}{2} \iint\left[N_{x}\left(\frac{\partial w}{\partial x}\right)^{2}+N_{y}\left(\frac{\partial w}{\partial y}\right)^{2}+2 N_{x y} \frac{\partial w}{\partial x} \frac{\partial w}{\partial y}\right] d x d y \\
\Delta U=\frac{D}{2} \iint\left\{\left(\frac{\partial^{2} w}{\partial x^{2}}+\frac{\partial^{2} w}{\partial y^{2}}\right)^{2}-2(1-v)\left[\frac{\partial^{2} w}{\partial x^{2}} \frac{\partial^{2} w}{\partial y^{2}}-\left(\frac{\partial^{2} w}{\partial x \partial y}\right)^{2}\right]\right\} d x d y \\
D=E t^{3} /\left[12\left(1-\nu^{2}\right)\right]
\end{gathered}
$$

where $\Delta T$ stands for the work done by external forces, $\Delta U$ for the plate's strain energy, $N_{x}$ for the plate force in $x$ direction, $N_{y}$ for the plate force in $y$ direction, $N_{x y}$ for the shear force, $w(x, y)$ for the deformation of the plate in local $z$ direction, $\nu$ for the Poisson's ratio of the material, $E$ for the Young modulus of the material and $t$ for the thickness of the analysed plate.

\section{THE ELASTIC LOCAL BUCKLING OF SIMPLY SUPPORTED BEAM'S FLANGE}

The models of flange used were of the following parameters: $L$ of $600,1000,1500$ and $2000 \mathrm{~mm}, b_{f}$ of 25,50 and $100 \mathrm{~mm}, t_{f}$ of 2 and $7 \mathrm{~mm}$ for $b_{f}$ of 25 and $100 \mathrm{~mm}, t_{f}$ of 2, 2.5, 3, $3.5,4,5,6,7,8$ and $10 \mathrm{~mm}$ for $b_{f}$ of $50 \mathrm{~mm}$.

The solution for the fixed flange is based on the shape of buckling mode as given in (5), $\delta_{z}=\varphi_{x}=0$ along the long edge (Figure 1). The solution depends on values of the $m$ and $n$ parameters, related to the plate's aspect ratio $L / b_{f}$ and loading conditions - either PL or UDL. The shape of the eigenform is checked against the finite element analyses (hereinafter: FEA) output.

$$
w(x, y)=(x / L)^{m} \sin (n \pi x / L) \sin (\pi x / L)^{2}\left(y / b_{f}\right)^{2}
$$




$$
\begin{array}{ll}
\sigma_{c r, f f i x}=E t_{f}^{2}\left[\alpha_{1} L^{4}+\alpha_{2} L^{2} b_{f}{ }^{2}(1-1.5 \nu)+\alpha_{3} b_{f}^{4}\right] /\left[100000 L^{2} b_{f}^{4}\left(1-\nu^{2}\right)\right] \\
\alpha_{1, \mathrm{PL}}=50000\left(b_{f} / L\right)^{2} & \alpha_{1, \mathrm{UDL}}=30000\left(b_{f} / L\right)^{2} \\
\alpha_{2, \mathrm{PL}}=65000+500000\left(b_{f} / L\right) & \alpha_{2, \mathrm{UDL}}=65000+500000\left(b_{f} / L\right) \\
\alpha_{3, \mathrm{PL}}=600000+17500\left(L / b_{f}\right)^{2} & \alpha_{3, \mathrm{UDL}}=700000+25000\left(L / b_{f}\right)^{2}
\end{array}
$$

For all the solutions, despite of the plate's aspect ratio, the final solution takes form of (6), where $\sigma_{c r, f f i x}$ stands for the elastic buckling stress of the plate. The value of parameters $\alpha_{1}, \alpha_{2}$ and $\alpha_{3}$ depends on the parameters described above. The values received from closed form $(\mathrm{CF})$ solutions of (6) are presented in Figure 3 with markers. Plots of proposed (7-9) are given as well using dotted and dashed lines.

In (6), parameters $\alpha_{1}$ and $\alpha_{2}$ are used as the multipliers of the $L^{4}$ and $L^{2} b_{f}{ }^{2}$, respectively. As the length of the plate is used, parameters are convergent to the certain value. In case of $\alpha_{1}$, the parameter can be treated as constant when $L / b_{f}$ is over 30 , in case of $\alpha_{2}$ (less dependent on $L$ ), the parameter stabilises with $L / b_{f}$ over 50 . This is not the case for $\alpha_{3}$, which is a multiplier for the $b_{f}{ }^{4}$. For the estimation of the equation the least squares method was used, with the additional restriction that $\alpha_{1}, \alpha_{2}$ and $\alpha_{3}$ must always be positive.

The identical procedure is implemented for the pinned flange model. Varying assumptions and results are given below in (10-13). Note that $\sigma_{c r, f, p i n}$ stands for the elastic buckling stress of the plate. Results from FEA models of plates can be replicate by proposed (12 and 13).

$$
\begin{gathered}
w(x, y)=(x / L)^{m} \sin (\pi x / L)^{2}\left(y / b_{f}\right) \\
\sigma_{c r, f, p i n}=E t_{f}^{2}\left[\alpha_{4} L^{2}(1-\nu)+\alpha_{5} b_{f}^{2}\right] /\left[100 L^{2} b_{f}^{2}\left(1-\nu^{2}\right)\right] \\
\alpha_{4, \mathrm{PL}}=110\left(b_{f} / L\right)^{0.2} \quad \alpha_{4, \mathrm{UDL}}=100\left(b_{f} / L\right)^{0.2} \\
\alpha_{5, \mathrm{PL}}=6500\left(b_{f} / L\right)^{0.8} \quad \alpha_{5, \mathrm{UDL}}=7500\left(b_{f} / L\right)^{0.8}
\end{gathered}
$$

a)

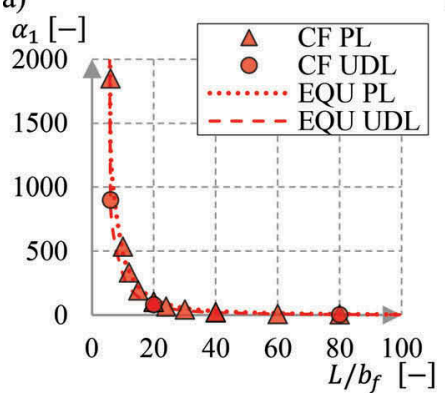

b)

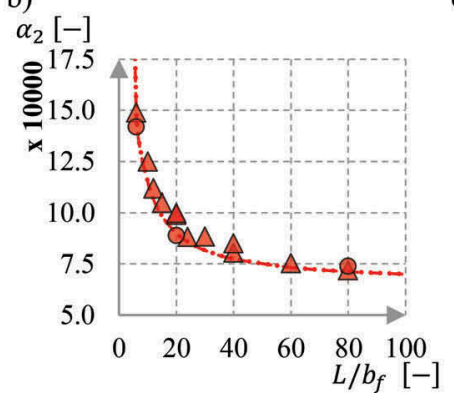

c)

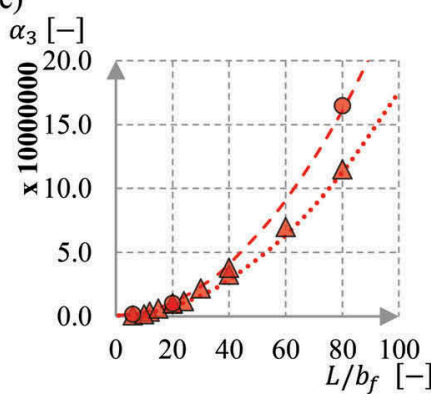

Figure 3. Equation parameters $\alpha_{1}, \alpha_{2}$, and $\alpha_{3}$ against $L / b_{f}$ ratio. a) $\alpha_{1}$ parameter, b) $\alpha_{2}$ parameter, c) $\alpha_{3}$ parameter. 


\section{THE ELASTIC LOCAL BUCKLING OF SIMPLY SUPPORTED BEAM'S WEB}

The models of web used were of the following parameters: $L$ of $600,1000,1500$ and $2000 \mathrm{~mm}$, $h_{w}$ of 100,200 and $400 \mathrm{~mm}, t_{w}$ of 2.2 and $5.2 \mathrm{~mm}$ for $h_{w}$ of 100 and $400 \mathrm{~mm}, t_{w}$ of 2.2, 2.7, 3.2, 3.7, 4.2, 4.7 and $5.2 \mathrm{~mm}$ for $h_{w}$ of $50 \mathrm{~mm}$. For all the models the $A_{f}$ was varying between 0 , $50,100,200,250,300,400,500,1000,2000,4000,5000,7500$ and $10000 \mathrm{~mm}^{2}$.

The influence of the flange onto the behaviour of the web is not limited to BC only. For the significant flange area $A_{f}$ the additional shear stress caused by tension/compression pair of forces in flanges influences the critical buckling strength of the web.

The method proposed is similar to the one presented in preceding section. The following solution does not consider the influence of additional stress caused by tension/compression in flanges. $\sigma_{c r, w, f i x, n s}$ stands for the elastic strength of the fixed web with shear flow disregarded.

$$
\begin{aligned}
& w(x, z)=(x / L)^{m} \sin (n \pi x / L) \sin (\pi x / L)^{2}\left(z^{2} / b_{f}\right) \sin \left(\pi z / b_{f}\right)^{2} \\
& \sigma_{c r, w, f i x, n s}=E t_{w}{ }^{2}\left(\beta_{1} L^{4}+\beta_{2} L^{2} h_{w}{ }^{2}+\beta_{3} h_{w}{ }^{4}\right) /\left[1000 L^{2} h_{w}{ }^{4}\left(1-\nu^{2}\right)\right] \\
& \beta_{1, \mathrm{PL}}=13500\left(h_{w} / L\right)^{2} \beta_{1, \mathrm{UDL}}=9500\left(h_{w} / L\right)^{2} \\
& \beta_{2, \mathrm{PL}}=10000+25000\left(h_{w} / L\right)^{2} \beta_{2, \mathrm{UDL}}=9000+22500\left(h_{w} / L\right)^{2} \\
& \beta_{3, \mathrm{PL}}=37500+9500\left(L / h_{w}\right)^{2} \beta_{3, \mathrm{UDL}}=23500+6000\left(L / h_{w}\right)^{2}
\end{aligned}
$$

The solution for the pinned web takes a similar form to the solution for the fixed web. Analyses of the elastic buckling strength of models with various BC and loading conditions lead to proposal of the reduction factor, $\chi$, which can be applied to the Equation (15) in order to obtain the elastic buckling strength of the pinned web. Equations (19-21) propose the $\chi$ factor and the resultant $\sigma_{c r, w, p i n, n s}$, critical elastic strength of the pinned web with shear flow disregarded.

$$
\begin{gathered}
\chi_{\mathrm{PL}}=1.55+0.08\left(L / h_{w}\right)^{0.3}-550\left(t_{w} / h_{w}\right)^{3} \\
\chi_{\mathrm{UDL}}=2.65-0.10\left(L / h_{w}\right)^{0.3}-1250\left(t_{w} / h_{w}\right)^{3} \\
\sigma_{c r, w, p i n, n s}=\sigma_{c r, w, f i x, n s} / \chi
\end{gathered}
$$

The consideration of the shear flow is based upon the assumption that the buckling mode can be either a bending buckling, coupled buckling or a shear buckling. In case of web with no shear flow the bending buckling takes place, changing to different forms as the $A_{f}$ rises. (22) shows the relation between the maximal bending and shear stress in the web with no shear flow, $\vartheta_{n s}$. Similarly, $\vartheta$ is a bending stress to shear stress relation with shear flow considered (23). The shear flow reduction factor $\psi$ is proposed based upon the limitation of bending stress (in the form of upper limitation of 1) and balance between shear stress and shear flow dictated by aspect ratio dependent parameters $\alpha_{\psi}$ and $\beta_{\psi}$, see (24-26). (27) shall be used for determination of the buckling strength of the web for both PL and UDL loading scenarios.

$$
\begin{array}{cc}
\vartheta_{n s, \mathrm{PL}}=h_{w} /(4 L) & \vartheta_{n s, \mathrm{UDL}}=h_{w} /(2 L) \\
\vartheta_{\mathrm{PL}}=\left(A_{f} / t_{w}+h_{w} / 4\right) / L & \vartheta_{\mathrm{UDL}}=\left(2 A_{f} / t_{w}+h_{w} / 2\right) / L \\
\psi=\min \left(1 ;\left[\alpha_{\psi}+\beta_{\psi}\left(\vartheta / \vartheta_{n s}\right)\right]\right)
\end{array}
$$




$$
\begin{gathered}
\psi_{\mathrm{PL}}=\min \left(1 ;\left\langle 0.7 \sqrt{h_{w} / L}+0.7\left(L / h_{w}\right)\left\{h_{w} /\left[4\left(A_{f} / t_{w}+h_{w} / 4\right)\right]\right\}\right\rangle\right) \\
\psi_{\mathrm{UDL}}=\min \left(1 ;\left\langle 0.7 \sqrt{h_{w} / L}+0.7\left(L / h_{w}\right)\left\{h_{w} /\left[2\left(A_{f} / t_{w}+h_{w} / 2\right)\right]\right\}\right\rangle\right) \\
\sigma_{c r, w, f i x}=\sigma_{c r, w, f i x, n s} * \psi \quad \sigma_{c r, w, p i n}=\sigma_{c r, w, p i n, n s} * \psi
\end{gathered}
$$

Results from FEA (markers) and proposed equations (lines) are shown in Figure 4 for the 1000/200/2.2 PL series.

\section{THE ELASTIC LOCAL BUCKLING CAPACITY OF SIMPLY SUPPORTED BEAM}

The procedure of strength approximation of semi-fixed plate (see Figure 2) is as given. The plate is assumed to be restrained along long edges with a rotational spring. Flange models are restrained with a spring of $K_{w}$ - stiffness corresponding to web stiffness per unit length whilst web models are restrained with $K_{f}(28)$. Based on the relation between the stiffnesses of plate and the restraint, the fixity factors for flange and web $\left(\gamma_{f}\right.$ and $\left.\gamma_{w}\right)$ are proposed (29 and 30). As $\gamma$ varies between 0.0 and 1.0, the buckling mode of the segment varies between the pinned and fixed eigenform. The segment's capacity is as in (31).

Final equation (32) estimates the buckling capacity of the whole beam. The results of (31) are compared and the lower-bound value is assumed as the buckling strength of the whole beam. Figure 5 presents the comparison of (31 and 32) against results of FEA. Models of the

a)

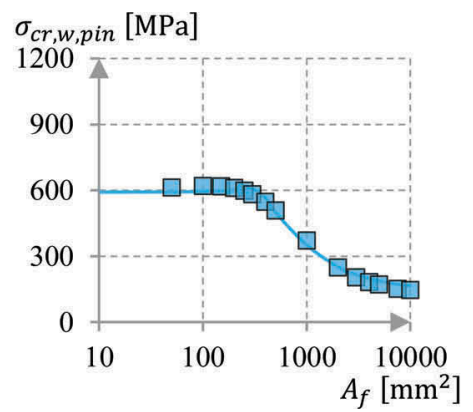

b)

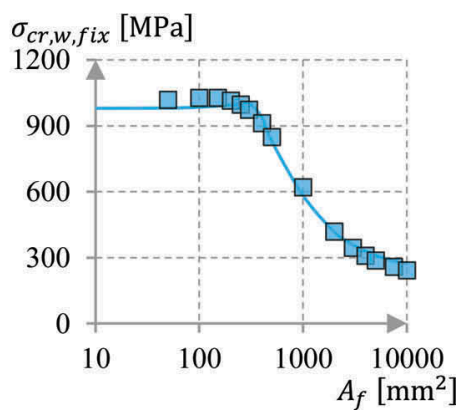

Figure 4. The strength reduction due to the shear flow effects. a) pinned web, b) fixed web.

a)

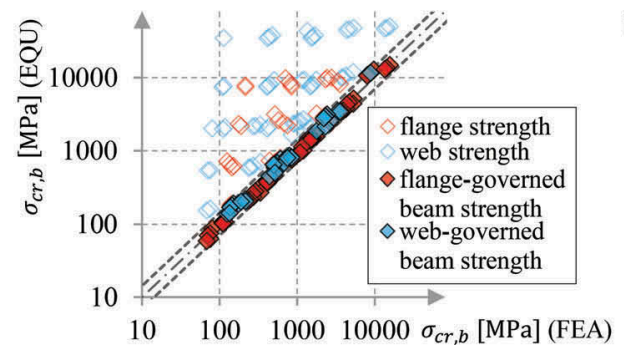

b)

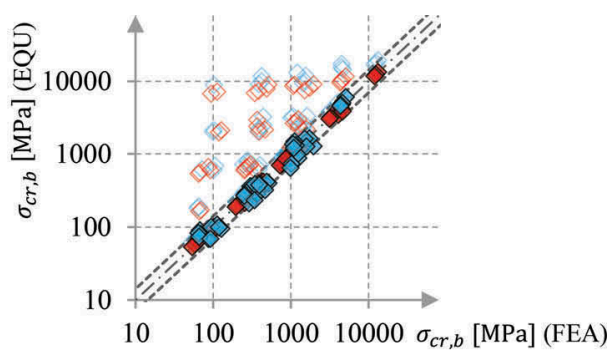

Figure 5. Comparison of results from proposed equations (EQU) and numerical analyses (FEA). a) PL: $\sigma_{c r}$ of the flange, web and beam (EQU) against $\sigma_{c r}$ of the beam (FEA), b) UDL: $\sigma_{c r}$ of the flange, web and beam (EQU) against $\sigma_{c r}$ of the beam (FEA). 
beams were built of segments having the same dimensions as in sections 2 and 3 . All models were consisting of S4R5 elements (12 for each flange, 22 for web, 180 length-wise) of certain thickness representing beam segments made of elastic material, with the $\mathrm{BC}$ and loads as shown in Figure 1. The FEA software used for the analyses was SOFiSTiK, with a crosscheck using Abaqus.

$$
\begin{gathered}
K_{w}=E t_{w}{ }^{3} /\left(2 h_{w}\right) K_{f}=E t_{f}{ }^{3} /\left(6 b_{f}\right) \\
\gamma_{f}=0<1.15 * \arctan \left\{1.75 *\left[-0.25-\log \left(K_{f} / K_{w}\right)\right]\right\} / \pi+0.5<1 \\
\gamma_{w}=0<1.15 * \arctan \left\{1.75 *\left[-0.25+\log \left(K_{f} / K_{w}\right)\right]\right\} / \pi+0.5<1 \\
\sigma_{c r, i}=\sigma_{c r, i, p i n}+\left(\sigma_{c r, i, f i x}-\sigma_{c r, i, p i n}\right) * \gamma_{i} \text { for } i \in(f ; w) \\
\sigma_{c r, b}=\min \left(\sigma_{c r, f} ; \sigma_{c r, w}\right)
\end{gathered}
$$

\section{SUMMARY}

This research is aimed to explain the elastic buckling behaviour of simply supported beam by fulfilling 2 targets: investigate the strength of the segments of the beam considering in-between interactions and to propose equation estimating the local buckling capacity of the whole beam.

It can be stated, that equations based upon the energy equilibrium are feasible for the estimation of the elastic bucking capacity of the simple plates building up the beam. It was proven that, by utilising equations for the estimation of the effects of the web-flange interactions, it is possible to approximate the elastic local buckling capacity of the beam with satisfying precision.

\section{ACKNOWLEDGMENTS}

This work was supported by the JST Program on Open Innovation Platform with Enterprises, Research Institute and Academia.

\section{REFERENCES}

Bradford, M.A., Hancock, G.J. 1984. Elastic Interactions of Local and Lateral Buckling in Beams, ThinWalled Structures, Applied Science Publishers Ltd, 2 (1983), pp. 1-25.

European Commission 2005. EN 1993-1-1 (2005) (English): Eurocode 3: Design of steel structures - Part 1-1: General rules and rules for buildings. European Committee for Standardization.

Fujak, S.M., Kimura, Y. 2019. A Comparison of The Capacity Evaluation Methods for Moment-Resistant Frames with Detailed Mid-Storey Pinned Connection as per Standards and Numerical Analyses with Respect to the Local Buckling of Members, 12th Pacific Structural Steel Conference, Tokyo, November 9-11, 2019.

Ikarashi, K. 2003. Buckling Strength of Simply Supported Web Plate Under the Action of Bending Shear Stress, Journal of Structural and Construction Engineering (Transactions of AIJ), Architectural Institute of Japan, 2003, Vol. 68, No. 565, pp. 135-141. (in Japanese)

Ikarashi, K., Suekuni, R., Shinohara, T., Wang, T. 2011. Evaluation of Plastic Deformation Capacity of H-shaped Steel Beams with Newly Proposed Limitation Value of Plate Slenderness, Journal of Structural and Construction Engineering (Transactions of AIJ), Architectural Institute of Japan, 2011, Vol. 76, No. 668, pp. 1865-1872. (in Japanese)

Kimura, Y., Suzuki, A., Kasai, K. 2019. Estimation of plastic deformation capacity for I-shaped beams with local buckling under compressive and tensile forces, Japan Architectural Review, 2019, 2:26-41.

Roberts, T.M., Jhita, P.S. 1983. Lateral, Local and Distortional Buckling of I-beams, Thin-Walled Structures, Applied Science Publishers Ltd, 1 (1983), pp. 289-308.

Timoshenko, S.P., Gere, J.M. 1961. Theory of Elastic Stability, Dover Publications Inc. 